\title{
Peri conceptional association of the triad of hyperhomocsteinemia, hypothyroidism and impaired carbohydrate metabolism with recurrent pregnancy loss
}

\author{
Bhagyashree Mallinath Gadwal ${ }^{1 *}$, Revathi S. Rajan², Kamini A. Rao ${ }^{3}$
}

\author{
${ }^{1}$ Department of Obstetrics and Gynacology, Mahadevappa Rampure Medical College, Kalaburagi, Karnataka, India \\ ${ }^{2}$ Mirror Health, Bengaluru, Karnataka, India \\ ${ }^{3}$ Milann The Fertility Centre, Bengaluru Karnataka, India
}

Received: 28 June 2021

Accepted: 26 July 2021

\author{
*Correspondence: \\ Dr. Bhagyashree Mallinath Gadwal, \\ E-mail: drbhagyasharan@gmail.com
}

Copyright: (c) the author(s), publisher and licensee Medip Academy. This is an open-access article distributed under the terms of the Creative Commons Attribution Non-Commercial License, which permits unrestricted non-commercial use, distribution, and reproduction in any medium, provided the original work is properly cited.

\begin{abstract}
Background: Recurrent pregnancy loss is a cause of great concern to the affected couple as well as the treating physician. We evaluated the periconceptional association of the triad of hyperhomocystienemia, hypothyroidism, and impaired carbohydrate metabolism with RPL and to identify the potential risk factors for RPL which are probably nutritional.

Methods: Seven hundred ninety-six consecutive pregnancies with two or more abortions referring to our Infertility clinic were selected to be studied in a descriptive, prospective observational study in 2012-2014. All the pregnant women were subjected to routine blood tests/ examination/ imaging as per hospital protocols. All underwent TSH, FT4, GTT $75 \mathrm{gm}$ (fasting and $2 \mathrm{~h}$ postprandial) as per routine antenatal workup. Additionally, fasting plasma homocysteine, antinuclear antibody, anti phospholipid antibody, anti- thyroid peroxidase antibody tests were performed for patients with RPL. All the patients were studied for the triad of hypothyroidism, hyperhomocysteinemia and impaired carbohydrate metabolism.

Results: The incidence of RPL was found to be $5.65 \%$. Majority of women $(51.1 \%)$ were in the age group 30-34 years. Majority of the women $(55.6 \%)$ had 2 previous abortions. Majority of women $(57.7 \%)$ had conceived normally. Majority of women $(46.7 \%)$ had BMI $<25$. PCOS was reported in $28.9 \%$ women. HHcy, hypothyroidism and impaired carbohydrate metabolism was found in $78 \%, 73 \%$ and $73 \%$ women, respectively. The presence of two factors was reported in 60 to $65 \%$ women. $56 \%$ women reported the presence of the triad. About $7 \%$ of the patients underwent repeat abortion in second trimester of which $66.6 \%$ had the association of triad.

Conclusions: Investigating these three factors in patients with RPL would help in early recognition, monitoring, and aggressive surveillance, which will help prevent obstetric complications. Simple nutritional correction may allow for better maternal and fetal programming and appropriate risk modulation.
\end{abstract}

Keywords: Hyperhomocysteinemia, Hypothyroidism, Impaired glucose metabolism, Recurrent pregnancy loss

\section{INTRODUCTION}

The American society for reproductive medicine (ASRM) defines recurrent pregnancy loss (RPL) as the spontaneous loss of two or more pregnancies. ${ }^{1}$ About $15 \%$ of couples are affected by RPL, with significant consequences concerning their partnership and quality of life. ${ }^{2,3}$ Parental chromosomal anomalies, uterine structural anomalies, maternal thrombophilic disorders and antiphospholipid antibodies have been directly associated with RPL. However, in almost $50 \%$ of cases, the pathophysiology remains unknown. ${ }^{4}$ In our country, heritable thrombophilias are less common. ${ }^{5}$ Among the undetermined cases, elevation in total homocysteine 
(tHcy) levels (hyperhomocysteinemia; HHcy), hypothyroidism, impaired carbohydrate metabolism, or combinations of these three pathologic conditions have been described as playing a role in the pathogenesis of RPL. Homocysteine is a non-essential amino acid that can be converted into cysteine or recycled into methionine, an essential amino acid, with specific B vitamins. Homocysteine levels vary between men and women, with a normal range typically between 5 to 15 micromol/l. Hyperhomocysteinemia is when levels exceed 15 micromol/l. ${ }^{6}$ Hyperhomocysteinemia (HHcy) has been underlined as an emerging risk factor for several diseases such as arterial and/or venous thrombosis, adverse pregnancy outcome, congenital malformations and vascular dementia. ${ }^{7-12}$

Thyroid hormones are very crucial for foetal development. Thyroid hormone disorders and high thyroid peroxidase antibodies (TPO-Ab) levels disturb folliculogenesis, spermatogenesis, embryogenesis and fertilisation, supporting a vital role in pregnancy loss. ${ }^{13}$ Thyroid disorders can be either hypothyroidism or hyperthyroidism. Euthyroid women are defined as those having normal TSH $(0.1-2.5 \mu \mathrm{IU} / \mathrm{l})$ in first trimester. Subclinical hypothyroidism is defined as high TSH $(>3.0$ $\mu \mathrm{IU} / 1)$ in the presence of normal levels of free T4 (0.8-2.0 $\mathrm{ng} / \mathrm{dl})$. Overt hypothyroidism is defined as high TSH $(>3.0 \mu \mathrm{IU} / \mathrm{l})$ with low Free T4 $(<0.8 \mathrm{ng} / \mathrm{dl}) .{ }^{14}$

Abnormal carbohydrate metabolism during pregnancy, such as impaired glucose tolerance (IGT) and gestational diabetes (GDM), is a relatively frequent disease affecting $2-5 \%$ of all pregnancies. As with other maternal disorders associated with macrovascular dysfunction, abnormal carbohydrate metabolism is related to augmented maternal and fetal-neonatal morbidity. ${ }^{15}$ Current study aims to evaluate the periconceptional association of the triad of hyperhomocystienemia, hypothyroidism, and impaired carbohydrate metabolism with RPL and to study the potential risk factors for RPL which are probably nutritional.

\section{Aim}

Aim of the current study was to evaluate the periconceptional association of the triad of hyperhomocystienemia, hypothyroidism, and impaired carbohydrate metabolism with RPL.

\section{METHODS}

Seven hundred ninety-six consecutive pregnancies with two or more abortions referring to Milann the fertility centre, Bangalore, India were selected to be studied in a descriptive, prospective observational study in 20122014. All the pregnant women were subjected to routine blood tests/ examination/ imaging as per hospital protocols. All underwent TSH, FT4, GTT 75 gm (fasting and $2 \mathrm{hr}$ postprandial) as per routine antenatal workup. Additionally, fasting plasma homocysteine, antinuclear antibody (ANA), anti phospholipid antibody (APLA), anti thyroid peroxidase antibody tests were performed for patients with RPL. All the patients were studied for the triad of hypothyroidism, hyperhomocysteinemia and impaired carbohydrate metabolism.

\section{Statistical analysis}

The case records were analyzed systematically for the above-mentioned parameters and clinical outcomes.

\section{RESULTS}

The incidence of RPL in current study was $5.65 \%$. The maximum numbers of women $(51.1 \%)$ were in the age group 30-35 years. The maximum number of women $(55.6 \%)$ had two abortions. The maximum number of women $(57.77 \%)$ had conceived normally. A maximum number of women (46.7 \%) had low BMI. $28.9 \%$ of RPL cases also had the presence of PCOS. The distribution of patients according to the presence of specific antibodies and anomalies is shown in (Table 7). The distribution of patients according to the presence of hypothyroidism, hyperhomocysteinemia, impaired carbohydrate metabolism or a combination thereof are as shown in (Table 8).

Table 1: The incidence of RPL.

\begin{tabular}{|lll|}
\hline Incidence of RPL & $\mathbf{N}$ & $\%$ \\
\hline Total RPL & 45 & 5.65 \\
\hline Other than RPL & 751 & 94.34 \\
\hline Total pregnancies & 796 & 100 \\
\hline
\end{tabular}

Table 2: The age-wise distribution of RPL cases.

\begin{tabular}{|lll|}
\hline Age group (years) & $\mathbf{N}$ & $\%$ \\
\hline $\mathbf{2 0 - 2 4}$ & 4 & 8.9 \\
\hline $\mathbf{2 5 - 2 9}$ & 11 & 24.4 \\
\hline $\mathbf{3 0 - 3 4}$ & 23 & 51.1 \\
\hline $\mathbf{3 5 - 3 9}$ & 5 & 11.1 \\
\hline $\mathbf{4 0 - 4 4}$ & 2 & 4.4 \\
\hline Total & 45 & 100.0 \\
\hline
\end{tabular}

Table 3: Distribution of subjects by the number of abortions.

\begin{tabular}{|lll|}
\hline No. of Abortions & $\mathbf{N}$ & $\%$ \\
\hline $\mathbf{2}$ & 25 & 55.6 \\
\hline $\mathbf{3}$ & 14 & 31.1 \\
\hline $\mathbf{4}$ & 5 & 11.1 \\
\hline $\mathbf{6}$ & 1 & 2.2 \\
\hline Total & 45 & 100 \\
\hline
\end{tabular}

\section{DISCUSSION}

The incidence of RPL in our study was found to be 5.65 $\%$. Globally, RPL is reported to occur in $0.5 \%-1 \%$ of 
total pregnancies. ${ }^{16,17}$ The incidence is high in our study as the data has been collected from an Infertility Centre frequented by couples already suffering from infertility and pregnancy loss. In current study, $46.7 \%$ of women had $\mathrm{BMI}<25,35.6 \%$ had BMI between 26 to 29, and $15.6 \%$ had $\mathrm{BMI}>30$. Both undernourishment and high BMI are associated with infertility, including recurrent pregnancy loss (RPL).

Table 4: Distribution of RPL pregnancies by mode of conception.

\begin{tabular}{|l|l|l|}
\hline Mode of conception & $\mathbf{N}$ & $\%$ \\
\hline Normally Conceived & 26 & 57.77 \\
\hline Timed Intercourse & 5 & 11.11 \\
\hline IUI & 3 & 6.66 \\
\hline IVF/ET & 11 & 24.44 \\
\hline
\end{tabular}

Table 5: Distribution of subjects by BMI.

\begin{tabular}{|l|l|l|}
\hline BMI & N & $\%$ \\
\hline $\mathbf{0 - 2 5}$ & 21 & 46.7 \\
\hline $\mathbf{2 6 - 2 9}$ & 16 & 35.6 \\
\hline $\mathbf{3 0}$ and more & 7 & 15.6 \\
\hline Total & 45 & 100.0 \\
\hline
\end{tabular}

Table 6: Distribution of RPL subjects according to the presence of PCOS.

\begin{tabular}{|l|l|l|}
\hline Presence of PCOS & N & $\%$ \\
\hline Yes & 13 & 28.9 \\
\hline No & 32 & 71.1 \\
\hline Total & 45 & 100 \\
\hline
\end{tabular}

Table 7: Distribution of subjects by presence of condition.

\begin{tabular}{|ll|l|}
\hline Presence of condition & $\mathbf{N}$ & $\%$ \\
\hline $\begin{array}{l}\text { Anti-thyroid peroxidase } \\
\text { antibodies }\end{array}$ & 10 & 22.2 \\
\hline Anti nuclear antibodies & 8 & 17.8 \\
\hline $\begin{array}{l}\text { Anti phospholipid } \\
\text { antibodies }\end{array}$ & 8 & 17.8 \\
\hline Uterine anomalies & 5 & 11.1 \\
\hline Chromosomal anomalies & 0 & 0 \\
\hline
\end{tabular}

Pre-pregnancy underweight status was found to have a greater risk of preterm birth, low birth weight and intrauterine growth restriction. Having low BMI can affect the development of the placenta in pregnant women and affect the growth of the fetus due to a lack of nutrients. One of the significant pathophysiological changes in obesity is the distribution of excess weight and fat (adipocytes). The build-up of adipocytes is known to cause insulin resistance, which is responsible for the natural history of various diseases, particularly diabetes mellitus. Adipocytes also contribute to the escalation in reactive oxygen species (ROS) and pro-inflammatory cytokines. This phenomenon is responsible for the dysfunction of blood vessels, leading to coronary artery disease and stroke. Other possible mechanisms of recurrent pregnancy loss in women with obesity are disturbances in the hypothalamic-pituitary-gonadal hormonal axis, endometrial receptivity, oocyte quality, and inflammatory markers. ${ }^{18}$

Table 8: Distribution of subjects by condition.

\begin{tabular}{|l|ll|}
\hline Condition & N & $\%$ \\
\hline Hypothyroidism & 33 & 73 \\
\hline Hyper homocysteinemia & 35 & 78 \\
\hline Impaired carbohydrate metabolism & 33 & 73 \\
\hline Hypothyroidism+hyperhomocystenemia & 28 & 62.2 \\
\hline $\begin{array}{l}\text { Hypothyroidism+impaired } \\
\text { carbohydrate metabolism }\end{array}$ & 29 & 64.4 \\
\hline $\begin{array}{l}\text { Hyperhomocysteinemia+impaired } \\
\text { carbohydrate metabolism }\end{array}$ & 27 & 60 \\
\hline
\end{tabular}

In our study, $28.9 \%$ of RPL cases also had the presence of PCOS. Thus, the clinical association of RPL in polycystic ovarian syndrome (PCOS) is more than common. However, the incidence rate between PCOS and RPL remains uncertain due to its wide variation in different studies. The high prevalence of hypersecretion of luteinising hormone and obesity in the syndrome has been reported as a risk factor for spontaneous abortion. Several studies document a possible association between insulin resistance (IR) and HHcy, with the incidence of the latter being high in PCOS women. Recent reports attest the occurrence of hypofibrinolysis associated with high plasminogen activator inhibitor-1 (PAI-1) in women with PCOS as a cause of RPL. The effects of elevated PAI-1 may also be aggravated by elevated homocysteine, eventually causing thrombosis. Thus, PCOS involves numerous confounding factors that may contribute, individually or in combination, to thrombosis and ultimately lead to RPL. ${ }^{19}$ In current study, hyperhomocysteinemia (HHyc), hypothyroidism and impaired carbohydrate metabolism were reported in $73 \%$, $78 \%$ and $78 \%$ patients, respectively. In addition, a combination of two factors was reported in $60-65 \%$ of patients, and $56 \%$ of patients reported the presence of all three factors. A meta-analysis of studies between 1992 to 1999 by Nelen et al showed a significant association between HHcy and recurrent early pregnancy loss. More recently also, increasing evidence is available for the association of HHcy and RPL. ${ }^{2,3}$ Inherited conditions such as gene polymorphisms; i.e. cystathionine beta synthase (CBS) or methylenetetrahydrofolate reductase (MTHFR) have been involved in explaining the pathophysiology of HHcy. ${ }^{20,21}$ Acquired conditions such as folate and/or vitamin B6/B12 deficiencies due to dysregulation of their normal metabolism and/or low dietary intake are the modifiable causes of HHcy. ${ }^{22,23}$

Besides, recent studies have shown many non-enzymatic factors affecting homocysteine levels. ${ }^{6,8}$ Insulin inhibits 
hepatic CBS, an enzyme involved in the conversion of homocysteine to cystathionine, leading to elevated homocysteine levels. Insulin resistance seems to escalate the homocysteine levels. ${ }^{24}$ HHcy interferes with endometrial blood flow and vascular integrity, resulting in increased oxidative stress in vascular endothelium and early pregnancy loss. ${ }^{25}$

Hypothyroidism is common in pregnancy, and there is a statistically significant relationship of hypothyroidism with recurrent pregnancy loss in $<20$ weeks of gestation. ${ }^{25}$ The ASRM and ESHRE guidelines confirm the association between thyroid dysfunction and the risk of miscarriage. As per the guidelines, there is an emerging consensus that TSH values $>2.5 \mathrm{mU} / 1$ should be considered as an abnormal result in RPL patients. ${ }^{26}$ Glycemic control and insulin sensitivity are the most critical factors in reproductive pathophysiology. Impaired glucose tolerance, diabetes mellitus and insulin resistance (IR) have been long known to be linked to adverse reproductive outcomes, including infertility, miscarriages, and adverse pregnancy outcomes. Several studies have shown a biochemical and clinical association between miscarriage and poor glycemic control and IR. ${ }^{27}$ In normal pregnancy, maternal circulating glucose can be delivered to the fetus via the fetoplacental circulation but is not accompanied by maternal insulin, which cannot cross the placental barrier. The high maternal glucose levels in GDM provoke fetal hyperglycemia and reactive fetal hyperinsulinemia, which lead to endothelial dysfunction within the fetal micro-and macrocirculation. ${ }^{28}$ Thus, the triad of hypothyroidism, HHcy and impaired carbohydrate metabolism are interlinked and together contribute to RPL. In our cohort, three patients, i.e. $6.91 \%$ of patients, underwent repeat abortion in the second trimester, of which two patients reported the presence of triad.

\section{CONCLUSION}

Investigating these three factors in patients with RPL would help in early recognition, monitoring, and aggressive surveillance, which will help prevent obstetric complications. Simple nutritional correction may allow for better maternal and fetal programming and appropriate risk modulation. By proper guidance to our adolescent cohort, we can also help in achieving the safe future reproductive life. More extensive studies are required for the extrapolation of the results.

\section{Funding: No funding sources}

Conflict of interest: None declared

Ethical approval: The study was approved by the Institutional Ethics Committee

\section{REFERENCES}

1. Penzias A, Bendikson K, Falcone T, Hansen K, hill M, Hurd W, et al. Definitions of infertility and recurrent pregnancy loss: a committee opinion. Fertil Steril. 2020;113(3):533-5.

2. Vomstein K, Feil K, Strobel L, Aulitzky A, HoferTollinger S, Kuon R. Immunological Risk Factors in Recurrent Pregnancy Loss: Guidelines Versus Current State of the Art. J Clin Med. 2021;10(4):869.

3. Rai R, Regan L. Recurrent miscarriage. Lancet. 2006;368:601-11.

4. Lee R, Silver R. Recurrent pregnancy loss: summary and clinical recommendations. Semin Reprod Med. 2000;18:433-40.

5. Qublan H, Eid S, Ababneh H, Amarin Z, Smadi A. Acquired and inherited thrombophilia: implication in recurrent IVF and embryo transfer failure. Hum Reprod. 2006;21:2694-8.

6. Veeranki S, Gandhapudi S, Tyagi S. Interactions of hyperhomocysteinemia and $\mathrm{T}$ cell immunity in causation of hypertension. Can J Physiol Pharmacol. 2017; 95(3):239-46.

7. Martinelli I. Risk factors in venous thromboembolism. Thromb Haemost. 2001;86:395403.

8. Fatini C, Gensini F, Battaglini B, Prisco D, Cellai A, Fedi S. Angiotensin convertine enzyme DD genotype, angiotensin type 1 receptor CC genotype, and hyperhomocysteinemia increase first-trimester fetalloss susceptibility. Blood Coagul Fibrinol. 2000;11: 657-62.

9. Wouters M, Boers G, Blom H, Trijbels F, Thomas C, Borm G. Hyperhomocysteinemia: a risk factor in women with unexplained recurrent early pregnancy loss. Fertil Steril. 1993;60:820-5.

10. Steegers-Theunissen R, Boers G, Trijbel F, Finkelstein J, Blom H, Thomas C, et al. Maternal hyperhomocysteinemia: a risk factor for neural-tube defects? Metabolism. 1994;43:1475-80.

11. Bertsch T, Mielke O, Holy S, Zimmer W, Casarin W, Aufenanger J, et al. Homocysteine in cerebrovascular disease: an independent risk factor for subcortical vascular encephalopathy. Clin Chem Lab Med. 2001;39:721-4.

12. Hermann W, Knapp J. Hyperhomocysteinemia: a new risk factor for degenerative diseases. Clin Lab. 2002;48:471-81.

13. Sultana S, Nallari P, Ananthpur V. Recurrent Pregnancy Loss (RPL): An overview. J Women's Heal Dev. 2020;3:302-15.

14. Verma P, Roy D. Hypothyroidism and early pregnancy loss: an overview. Int J Reprod Contracept Obstet Gynecol. 2020;9(12):5065.

15. Paradisi G, Biaggi A, Ferrazzani S, S C, Caruso A. Abnormal Carbohydrate Metabolism During Pregnancy. Diabetes Care. 2002;25:560-4.

16. Baek K, Lee E, Kim Y. Recurrent pregnancy loss: the key potential mechanisms. Trends Mol Med. 2007;13(7):310-7.

17. Porter T, Scott J. Evidence-based care of recurrent miscarriage. Best Pract Res Clin Obstet Gynaecol. 2005;19(1):85-101. 
18. Utami T, Wiyono L, Muskitta N, Soerojo N, Sumarsudi K, Felicia I. The Risk of recurrent pregnancy loss in patients with a high body mass index: an evidence-based case report. Pak $\mathbf{J}$ Nutr. 202;19(10):460-7.

19. Chakraborty P, Goswami S, Rajani S, Sharma S, Kabir S, Chakravarty B, et al. Recurrent Pregnancy Loss in Polycystic Ovary Syndrome: Role of Hyperhomocysteinemia and Insulin Resistance. PLoS One. 2013;8(5):e64446.

20. Mudd S, Skovby F, Levy H, Pettigrew H, Wilcken B, Pyeritz R, et al. The natural history of hoomocystinuria due to cystathionine beta-synthase deficiency. Am J Hum Genet. 1985;37:1-31.

21. Harmon D, Woodside J, Jarnell J, McMaster D, Young I, McCrumm E, et al. The common 'thermolabile' variant of methylene tetrahydrofolate reductase is a major determinant of mild hyperhomocysteinaemia. QJM. 1996;89:571-7.

22. Kang S, Wong P, Norusis M. Homocysteinemia due to folate deficiency. Metabolism. 1987;36:458-62.

23. Ubbink J, van der Mewe A, Delport R, Allen R, Stabler S, Roezler R, et al. The effect of a subnormal vitamin B-6 status on homocysteine metabolism. J Clin Invest. 1996;98:177-84.

24. Tayebe H, Moghadami-Tabrizil N, Davari-Tanhal F, Salmanian B. High plasma homocysteine and insulin resistance in patients with polycystic ovarian syndrome. Iranian J Rep Med. 2011;9(3):223-8.
25. Kaur R, Gupta K. Endocrine dysfunction and recurrent spontaneous abortion: An overview. Int $\mathrm{J}$ Appl Basic Med Res. 2016;6(2):79-83.

26. Vomstein K, Feil K, Strobel L, Aulitzky A, HoferTollinger S, Kuon R, et al. Immunological Risk Factors in Recurrent Pregnancy Loss: Guidelines Versus Current State of the Art. J Clin Med. 2021;10(4):869.

27. Tamara T, Elkholy A, Elsaed N, Abdellatif M, Essam N, Selem M. Association between Unexplained Recurrent Miscarriage and Insulin Resistance. Int J Reprod Med Gynecol. 2018;4(1):1-5.

28. Echeverriaa C, Eltitb F, Santibanezd J, Gaticaf S, Cabello-Verrugiof C, Simon F. Endothelial dysfunction in pregnancy metabolic disorders. BBAMol Basis Dis. 2020;1866(2):52-9.

29. Nelen W, Steegers E, den Heijer M, Eskes T. Hyperhomocysteinemia and recurrent early pregnancy loss: a meta-analysis. Fertil Steril. 2000;74(6):1196-9.

Cite this article as: Gadwal BM, Rajan RS, Rao KA. Peri conceptional association of the triad of hyperhomocsteinemia, hypothyroidism and impaired carbohydrate metabolism with recurrent pregnancy loss. Int J Reprod Contracept Obstet Gynecol 2021;10:3549-53 